\title{
Observations of possible jet formation in the binary blazar OJ287
}

\author{
Helen Jermak ${ }^{1,2}$, Iain A. Steele ${ }^{1}$, Gavin P. Lamb ${ }^{1}$, Mauri Valtonen ${ }^{3}$ \\ and Staszek Zola ${ }^{4}$ \\ ${ }^{1}$ Astrophysics Research Institute, Liverpool John Moores University, \\ 146 Brownlow Hill, Liverpool, L3 5RF, UK. \\ email: i.a.steele@ljmu.ac.uk, g.p.lamb@2010.ljmu.ac.uk \\ ${ }^{2}$ Dept. of Physics, Lancaster University, Bailrigg Campus, \\ Lancaster, LA1 4YW, UK. \\ email: h.e.jermak@ljmu.ac.uk \\ ${ }^{3}$ FINCA, University of Turku, Väisäläntie 20, 21500 Piikkiö, Finland. \\ email:mvaltonen2001@yahoo.com \\ ${ }^{4}$ Jagiellonian University, Gołçbia 24, 31-007 Kraków, Poland. \\ email: szola@oa.uj.edu.pl
}

\begin{abstract}
The blazar, OJ287, underwent a predicted period of flaring in late 2015, triggering multiple observing campaigns to conduct intensive monitoring. We present the polarisation data taken during this event with the Ringo3 polarimeter on the Liverpool Telescope and discuss two possible causes of the flares.
\end{abstract}

Keywords. polarization, galaxies: active, galaxies: jets, accretion, accretion disks

\section{Introduction}

In November- December 2015, OJ287, the binary supermassive black holes system, went into predicted outburst; interpreted as the interaction of a supermassive black hole with the accretion disk of the primary supermassive black hole (Valtonen et al. 2016). 20 days after the initial flare, a second flare was observed, with a simultaneous high optical degree of polarisation flare.

\section{Overview}

The first flare at MJD 57360 (see Valtonen et al. (2016) for full light curve) is predominantly thermal and shows low degree of polarisation. Therefore, any of the typical non-thermal emission from the primary's jet is diluted by the increase in thermal emission from the impact of the secondary supermassive black hole (SMBH2) with the primary's accretion disk.

To explain the second, strongly polarised flare (at MJD 57380, see Figure 1) there must be an ordered magnetic field or magnetic reconnection. While it is possible that the polarisation flare is coincidentally from the primary jet in OJ287, it is not possible that this is caused by the impact due to light crossing times across the accretion disk. It may be coincidental that the primary jet flares shortly after the impact, however, the second strongly polarised peak is also present during the 1984 outburst which would suggest the events are connected (Smith et al. 1987). This may suggest the formation of a jet on the SMBH2 or magnetic reconnection occurring as the secondary approaches the primary. 


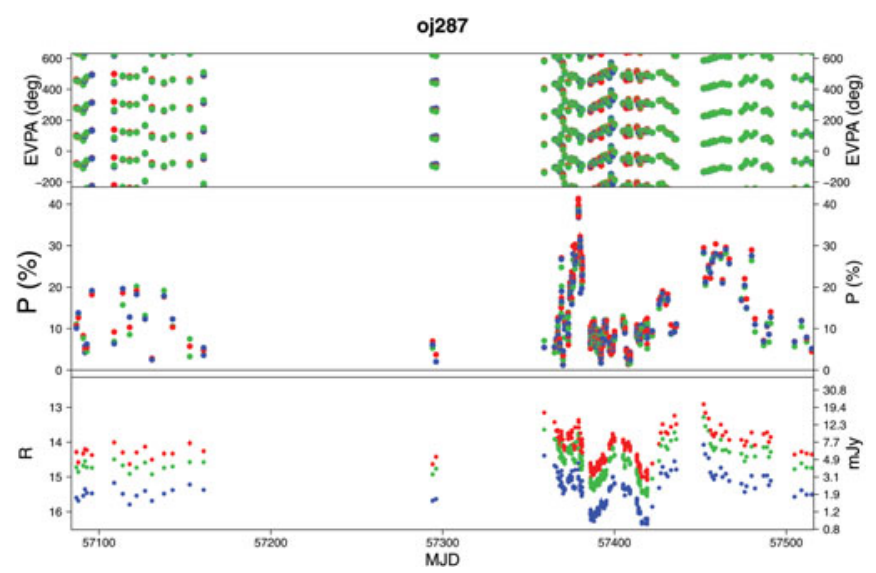

Figure 1. The multi-colour Ringo3 polarisation plots and light curve from $\mathrm{MJD}=$ 57100-57500. The blue, green and red points represent data from the respective Ringo3 cameras.

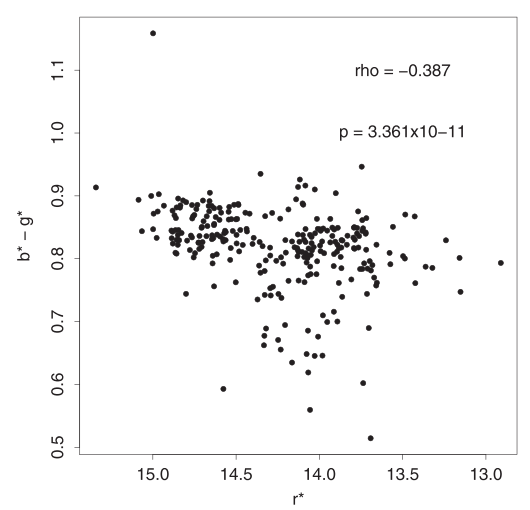

Figure 2. The colour magnitude plot for OJ287 using the Ringo3 bands $b^{*}, g^{*}$ and $\mathrm{r}^{*}$. The results from the Spearman Rank Correlation analysis are shown on the plot.

\subsection{Colour analysis}

The Ringo3 polarimeter measures photometry and polarimetry simultaneously at three wavelengths (Arnold et al. 2012). The three Ringo3 wavelength bands are: $\mathrm{r}^{*}$ ('red') = $770-1000 \mathrm{~nm}, \mathrm{~g}^{*}$ ('green') $=650-740 \mathrm{~nm}$ and $\mathrm{b}^{*}$ ('blue') $=350-640 \mathrm{~nm}$.

Figure 2 shows the $b^{*}-g^{*}$ colour plotted against the $r^{*}$ magnitude. The Spearman rank correlation coefficient, rho and the p value are shown on the plot and suggest a significant negative correlation of rho $=-0.387$ for OJ287 over the Ringo3 observing period. This correlation suggests that the source is 'bluer' when brighter, like many BL Lac-type blazars. The spectral properties of the source during the flaring period show significant negative correlations of a similar value. This suggests that the contribution from red light (i.e. jet emission) and blue light (accretion disk and/or high energy jet emission) does not significantly change during the impact.

\section{References}

Arnold, D. M., Steele, I. A., Bates, S. D., Mottram, C. J., \& Smith, R. J. 2012, in SPIE Conference Series, Vol. 8446, SPIE Conference Series

Smith, P. S., Balonek, T. J., Elston, R., \& Heckert, P. A. 1987, ApJS, 64, 459

Valtonen, M. J., Zola, S., Ciprini, S., et al. 2016, ApJL, 819, L37 